\title{
Coordinated multi-spacecraft observations of the Martian plasma environment
}

A community White Paper submitted to the Decadal Survey on Planetary Science and Astrobiology 2023 - 2032

\section{Corresponding author:}

Beatriz Sánchez-Cano University of Leicester, Leicester, United Kingdom +44 (0) 116252 3565, bscmdr1@leicester.ac.uk

Co-authors:

Majd Mayyasi

Kerstin Peter

Cesar Bertucci

Xiaohua Fang

Christopher M. Fowler

Zachary R. Girazian

Jingnan Guo

Maria Hamrin

Mika Holmberg

Bruce M. Jakosky

François Leblanc

Christina Lee

Mark Lester

Rob Lillis

Janet Luhmann

Yingjuan Ma

Christian Mazelle

Karim Meziane

Robin Ramstad

Shaosui Xu
Boston University, USA

RIU-Planetary Research, Germany

Inst. Astronomía, Física del Espacio, Argentina

University of Colorado Boulder, USA

University of California Berkeley, USA

University of lowa, USA

University of Science and Technology of China, China

Umeå University, Sweden

European Space Agency, Netherlands

University of Colorado Boulder, USA

LATMOS-CNRS, Sorbonne Université, France

University of California Berkeley, USA

University of Leicester, UK

University of California Berkeley, USA

University of California Berkeley, USA

UCLA, USA

IRAP, CNRS-University of Toulouse-CNES, France

University of New Brunswick, Canada

University of Colorado Boulder, USA

University of California Berkeley, USA

\section{Staunch Supporters:}

Nicolas Andre

David Andrews

Stas Barabash

Dolon Bhattacharyya

Stephen W. Bougher

David Brain

Marco Cartacci

Feng Chu

John Clarke

Thomas Cravens

Matteo Crismani

Shannon M. Curry

Gina A. DiBraccio

Chuanfei Dong

Yaxue Dong

Eduard Dubinin

Anders Eriksson

J. Tracy Esman
Scott Evans

Matthew O. Fillingim

Markus Fraenz

Rudy A. Frahm

Manuel Grande

Guillaume Gronoff

Gwen Hanley

Yuki Harada

Kim Konstantin

Andrew J. Kopf

Yuni Lee

Mike Liemohn

Paul Mahaffy

David L. Mitchell

Clara Narvaez

Hermann Opgenoorth

Roberto Orosei

Martin Pätzold

\author{
Gangkai Poh \\ Leonardo Regoli \\ Norberto Romanelli \\ Zhaojin Rong \\ Suranga Ruhunusiri \\ Shotaro Sakai \\ Alexander Shane \\ Sergey Shuvalov \\ Marek Slipski \\ Katerina Stergiopoulou \\ Shane W. Stone \\ Silvia Tellman \\ Scott Thaller \\ Ed Thiemann \\ Oleg Vaisberg \\ James Wild \\ Paul Withers \\ Roger Yelle
}




\section{Executive summary}

This White Paper discusses the key open science questions regarding the effects of the solar wind interaction at Mars. Understanding the Martian plasma system behavior as a whole (from the surface to space) should be a priority for space research as it holds significant clues to the evolution of Mars' climate and past/present habitability. Moreover, it plays a critical role for both unmanned and manned exploration.

\section{Our recommendations for the Committee for the Decadal Survey on Planetary Science and Astrobiology 2023 - 2032 are:}

1) Recognize the importance of multipoint space environment and upper atmosphere missions toward filling the knowledge gaps related to cause-and-effect that limit our knowledge of Mars and its history.

2) Deliver electric field, magnetic, ionospheric remote sensing, and radiation sensors to the surface with future landed missions.

3) Enable accommodation of solar and space environment monitoring instrument packages on future Mars orbiters (perhaps as a partnership of Heliophysics and Planetary Divisions).

4) Deliver novel platforms and instruments for making in situ observations, in particular of ion and neutral composition, at altitudes between 20 and $130 \mathrm{~km}$.

\section{Current state of knowledge and motivation}

The Martian space environment, which includes the solar wind, magnetosphere, ionosphere and atmosphere, is a complex system with simultaneous downward and upward couplings. Previous Mars missions, especially MAVEN and Mars Express, have shown this complex system is a key to fully understanding the evolution of Mars' climate, as well as its past/present habitability. This is especially important on the eve of the human exploration of the red planet as the plasma system plays a key role in the performance of communications and spacecraft systems, rover/lander radio operations.

Unlike most planets in our Solar System, Mars does not have a global magnetic field. The solar wind interacts directly with the upper atmosphere, inducing a magnetosphere. On the dayside, this interaction occurs within the ionosphere (ion and electron layer at $100-500$ $\mathrm{km})^{[1]}$ as can be seen in Figure 1 . As a result, the solar wind can strip away Mars' atmosphere very effectively ${ }^{[2]}$. The ionosphere-solar wind interaction is more complex over a region of the southern hemisphere where highly non-uniform crustal magnetic fields are located (Figure 1). These fields can interact directly with the solar wind producing a "hybrid magnetosphere", i.e. with features of both induced and intrinsic magnetospheres, which affect the whole planet because it changes as the crustal magnetic fields rotate with the planet $^{[3]}$. Moreover, these crustal fields play an important role in guiding plasma motion, producing hemispheric asymmetry in the magnetosphere, ionosphere, and ion escape ${ }^{[4]}$. 


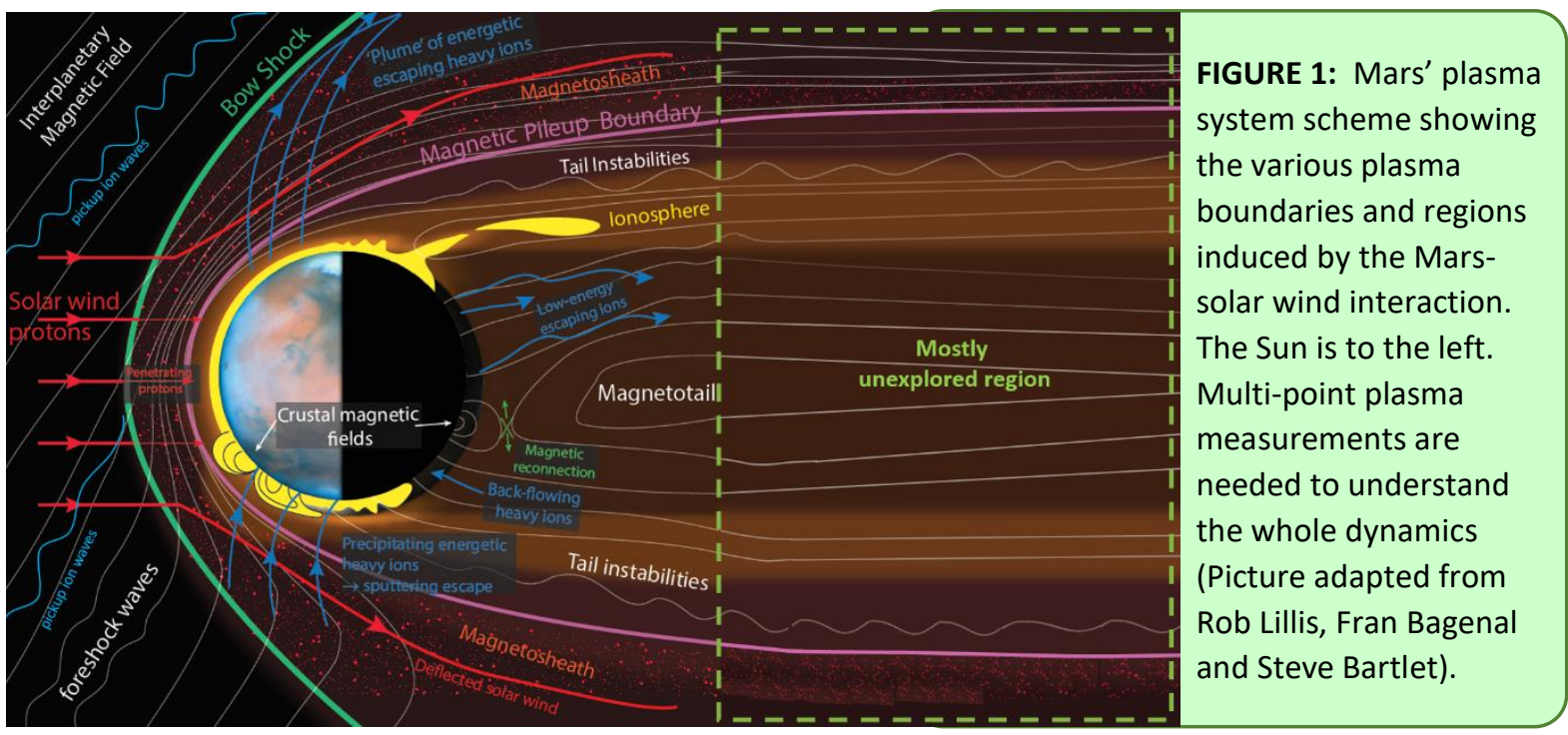

In addition, the neutral atmosphere is strongly coupled with the ionized part via collisions, being an important source of plasma variability. For example, Mars has strong lower atmospheric cycles (at the surface and lower-middle atmosphere) such as the water or $\mathrm{CO}_{2}$ cycles $^{[5]}$, as well as global dust storms ${ }^{[6,47]}$ and gravity waves ${ }^{[7]}$, that can contribute to enhancing escape processes ${ }^{[8]}$.

Despite the great progress in our understanding of the Martian plasma interaction and upper atmosphere system, thanks to nearly three decades of observations with MGS, Mars Express and MAVEN, each mission has left us with a new set of questions to answer. This paper will discuss three important and unanswered science topics related to Mars' interaction with the solar wind and its induced magnetosphere-ionosphere-atmosphere system.

Science gap 1: Coupled solar wind, magnetospheric, ionospheric and upper atmosphere dynamics investigations (multi point observational gaps)

Science gap 2: The lower ionosphere (observational gaps)

Science gap 3: Solar activity effects on Mars' system (observational gaps)

\section{Scientific potential of coordinated multi-point observations}

There have been several recent single spacecraft missions to Mars that have provided observations of the plasma environment, such as MGS, Mars Express, and MAVEN. They have enabled us to identify and characterize the basic properties of the various plasma boundaries and regions that exist in the Martian induced magnetosphere (e.g. the bow shock, magnetic pileup boundary, ionopause $\left.{ }^{[9,10,17]}\right)$. However, synergetic and simultaneous in-situ and remote-sensing multi-point measurements of the system are required to fully understand how these boundaries and regions respond to changes in the upstream solar wind and solar EUV conditions (the two main energy inputs to the system). Multi-point coordinated measurements are also required to determine how energy flows through the induced magnetosphere and into the ionosphere-atmosphere, causing important dynamics and energization.

We can draw on 60 years of space exploration at Earth, where multi-point missions (e.g., Cluster, THEMIS, Swarm, MMS) have revolutionized our understanding of the terrestrial solar 
wind-magnetosphere-ionosphere coupling. At Mars, "ad-hoc" multi-spacecraft studies between existing individual assets have been undertaken and have demonstrated the huge potential that a coordinated mission has ${ }^{[11,12]}$, but the instrument suites of existing assets are not designed for multi-point observations and opportunities for such analyses are rare. Thus, a dedicated mission that makes coordinated and simultaneous observations at different parts of the Martian system is critical to unravel the key mechanisms that strongly couple its surface, lower, mid and upper atmosphere, ionosphere, exosphere, induced magnetosphere and the solar wind (Figure 1). Specifically, the global response of the system to solar wind variability requires at least two well-separated spacecraft (one in the solar wind and the other inside the system). For local plasma dynamics, at least two spacecraft close to each other are required. In both cases, they will:

(i) Separate spatial variability from temporal variability

(ii) Capture variations on short spatial/temporal scales that cannot be resolved from a single spacecraft.

\section{SCIENCE GAP 1: Coupled solar wind, magnetospheric, ionospheric and upper atmosphere dynamics investigations}

\subsection{Solar wind control and role of crustal fields on the induced magnetosphere, plasma boundaries and currents.}

The solar wind interaction with the Martian system leads to the formation of several plasma boundaries, with the bow shock the outmost layer. Downstream of the bow shock, the incoming solar wind is decelerated, and the interplanetary magnetic field is compressed forming the induced magnetosheath region. The fundamental nature of the plasma boundaries, as formed by systems of currents coupling the solar wind, magnetosphere, and ionosphere, is not well understood. Electrical currents are a natural connection between different regimes within a planet plasma interaction system, as recently revealed at Mars for the first time with MAVEN observations ${ }^{[13]}$. Despite their importance, their spatial patterns, temporal variability, generation mechanisms, and full consequences are unknown at Mars. At the Earth, magnetospheric and ionospheric currents are coupled, and moreover, affect the upper neutral atmosphere in a feedback loop-especially during magnetic storms. In addition, the role of the crustal magnetic fields in controlling the near-Mars space environment, on both global and local scales, is still not well understood. Detailed and quantitative descriptions of the role(s) of currents and the crustal field throughout the ionosphere and magnetosphere as well as particle and energy exchanges between regions are still missing. These challenges require multi-point observations with higher data cadence covering upstream drivers and downstream responses and relating activities and variabilities among different space elements in the Mars' system.

\subsection{Neutral and ion escape rates}

Martian atmospheric losses to space today are mainly the result of thermal escape of neutral hydrogen and photochemical escape of neutral oxygen. These mechanisms, together with ion outflow, sputtering, and pickup ion escape, are believed to have led to the disappearance of liquid water on Mars over time ${ }^{[14,15]}$, a key point for habitability. However, the rates of the escaping neutral hydrogen and oxygen atoms cannot be directly measured with current 
technology due to their low density and energy (several eV). This means that we cannot observe what are thought to be the main atmospheric loss channels at Mars today ${ }^{[16]}$. The direct measurement of several $\mathrm{eV}$ neutral atoms is a technical challenge that is only now being addressed. To measure neutral escape should be a high priority for the next decade. Furthermore, Space Weather events (i.e., solar storms) greatly enhance the escape rate of water-originating species from Mars ${ }^{[18,19,20,21,43]}$. However, in situ ionospheric observations from individual spacecraft are limited to a single swath every few hours, making it difficult to determine the large-scale response of the ionosphere to dynamic space weather events, which have time scales ranging from minutes to days ${ }^{[44]}$. At Earth, decades of 'upstream solar wind monitor' data have provided unprecedented knowledge of the coupling between the solar wind and the magnetosphere-ionosphere system. The sparse solar wind coverage at Mars has greatly limited analogous understanding of the Mars system, and multi-point observations are needed to resolve such issues (see below).

In addition, the magnetotail may play an important role in ion escape processes through IMF reconnection with the crustal fields, similar to what happens at Earth during substorms ${ }^{[22,42]}$. However, because of the limited spatial sampling of individual spacecraft, we do not know the full implications of the tail on ion escape rates, or have adequate knowledge of how it varies under different conditions (e.g., different solar activity levels). Moreover, no mission has travelled far enough into the tail (>3-4 Mars radii) (Figure 1), with the only exception of a few transits by Mars 4, and Mars $5^{[23,24]}$. A detailed knowledge of the structure and variability of the Martian magnetospheric tail is essential for mapping and understanding the full range of ion outflow (escaping ions) and inflow (including particle precipitation) mechanisms, as well as sub-ion scale processes.

\subsection{Day-to-night plasma transport}

Day-to-night transport of plasma is crucial for maintaining the post sunset nightside ionosphere. This region may be especially important for supplying the 'cold' ion escape from the nightside and in the tail, which is separate from the higher energy pickup ion 'plume' [48]. However, our knowledge of the transport processes is incomplete ${ }^{[25,26]}$. In particular, high time cadence measurements of neutral and ion winds are unobtainable with current assets. It is believed plasma transport should be strongly dependent on the solar cycle. However, its dependence with solar zenith angle at the different Martian hemispheres needs still to be quantified. Multi-point measurements of solar energy inputs and of the driven winds are necessary to fully characterize day-to-night plasma transport.

\subsection{Nightside precipitation and aurora formation far from crustal fields}

Thanks mainly to the MAVEN mission, we now know that particle precipitation occurs everywhere on the Martian nightside, and not only over crustal magnetic regions as observed by MGS and Mars Express. Energy deposition from particle precipitation can drive ionospheric structure (through additional ionization), which can be observed in the form of global diffuse aurora $^{[27]}$, as well as low-altitude ionospheric layers (below $100 \mathrm{~km}$ ) that disrupt HF radio operations for several days ${ }^{[28]}$. Terrestrial auroral phenomena are explained by the combination of direct cusp entry of the solar energetic particles, together with magnetospheric tail reconnection during which energized magnetospheric particles travel along closed magnetic field lines into the Earth's atmosphere ${ }^{[29]}$. However at Mars, these different mechanisms have not been confirmed, and perhaps may be related to both the 
magnetotail field topology and poorly understood tail and cusp processes related to the localized crustal magnetic field regions. As at the Earth, auroral energy deposition may play a role in producing the enhancements in ion escape observed during episodes of disturbed space weather (see below).

\section{SCIENCE GAP 2: The lower ionosphere}

\subsection{The bottom-side ionosphere}

Current understanding of Mars' ionosphere and thermosphere is largely informed by "topdown" observations, i.e. those made from spacecraft in orbit. This means that the bottomside ionosphere is scarcely sampled. No measurements of the Martian ionosphere have been made from the surface at low radio frequencies. Thus, a significant gap is present in our knowledge. Understanding both the structure and dynamics of the lower ionosphere, and its coupling with the neutral atmosphere, would be greatly advanced by ground-based measurements. The lower part from which we have electron density observations (although no ion composition) is the secondary layer of the ionosphere, which peaks at $\sim 100 \mathrm{~km}$. Single radio occultation observations indicate that this layer responds to solar flares in the same way as the $E$ region of the terrestrial ionospher ${ }^{[30]}$. However, flare effects on short time scales at low altitudes are hardly investigated due to a lack of measurements, while little is known of its dependence on crustal magnetic fields regions ${ }^{[31]}$. In addition, a sporadic third layer can sometimes be found at $70-110 \mathrm{~km}$ with radio science observations ${ }^{[32]}$. The origin of this sporadic layer is under debate, it has been attributed to both the influx of meteoroids by models $^{[33]}$ and precipitation of solar wind ions ${ }^{[45]}$. However, the composition of these features remains unknown, with the exception that the IUVS-MAVEN instrument found that at $\sim 75 \mathrm{~km}$ there is a permanent layer of $\mathrm{Mg}^{+[34]}$ that is too small to be the sole origin of the sporadic third layer. This region requires a systematic exploration using cross-link radiooccultations between orbiters ${ }^{[46]}$, and exploration from the ground using radio-sounding techniques.

\subsection{Impact to the plasma environment near the Martian surface}

Below $70 \mathrm{~km}$, indirect observations (i.e., from the lack of reflected signal from the surface with radar soundings) indicate that ionization is present on both the day and nightsides when solar storms hit the planet ${ }^{[28]}$. Moreover, the amount of radiation that reaches the surface is being measured by the Mars Science Laboratory mission ${ }^{[37]}$. Some authors have theorized that an ionized layer at $\sim 25-30 \mathrm{~km}$ caused by galactic cosmic rays should be present ${ }^{[39]}$. This GCR source, together with the solar UV and X-ray photons, are believed to ionize the neutral atmosphere and aerosols close to the ground and generate an electric field from the ground to the ionosphere ${ }^{[39,40]}$. This totally unexplored region requires a systematic exploration from the ground in conjunction with orbiter observations, and is highly relevant to ground operations and future Mars exploration.

\subsection{Lower ionosphere control of HF radio propagation}

The ionosphere has strong effects on radio propagation, such as absorption of HF radio signals crossing the lower ionosphere $(<90 \mathrm{~km})$ or ionospheric-induced scintillations and group delay effects that are potential risks for orbital network of communications and navigational satellites at Mars ${ }^{[35]}$. MAVEN has observed small-scale ionospheric irregularities that appear 
analogous to terrestrial ionospheric E-region structures, which are known to cause disruption to communication systems at Earth ${ }^{[36]}$. Radio propagation issues are specially important after solar storms hit Mars (i.e., Space Weather) because in contrast with Earth where HF malfunctions last only a few hours, at Mars they typically last several days or weeks. Recently, combined observations from MAVEN, Mars Reconnaissance Orbiter, Mars Express and modelling have shown that HF radio blackouts are caused by energetic electrons from the solar wind that penetrate up to $60 \mathrm{~km}$ and ionize the middle-atmosphere (mesosphere) of Mars ${ }^{[28]}$. However, these studies are still at an early phase of research, and a more systematic characterization of their formation and behavior are currently missing, mainly because this part of the ionosphere is mostly unexplored.

\section{SCIENCE GAP 3: Solar activity effects on Mars' system}

Space weather real-time forecast at Mars is currently very challenging because among other factors, it needs a continuous solar and solar wind-monitoring platform to provide timely and accurate Space Weather information. At the moment, the most available measurements of the Mars-facing Sun and upstream solar wind at Mars occur when Mars and Earth are in apparent opposition or perfectly aligned along the Parker spiral (once every two years), and Earth satellites can be used. The challenge arises when both planets are not close to each other, which happens for about a (terrestrial) year and half. In those situations, Mars does not have a sufficiently accurate monitor of the Sun and local space environment, therefore the analysis of Space Weather effects on the Martian environment can be extremely difficult. These must depend on solar and solar wind observations taken, in the best of the cases, in the hours before when the spacecraft was in the solar wind ${ }^{[44]}$. The arrival of MAVEN in 2014 has improved our capability to monitor solar activity ${ }^{[41]}$, in part due to its comprehensive aeronomy instrumentation suite. However, MAVEN does not sample the solar wind all the time, meaning that assumptions and proxies must be used during times where solar wind observations are not present. Moreover, the orbital period of MAVEN changed in 2019 after an aerobraking campaign, and with that change, the orbit's apoapsis was reduced. Consequently, MAVEN is now taking less in-situ solar wind data than before.

Continuous in-situ solar wind and Space Weather observations are extremely important, together with simultaneous atmospheric observations, for most of the science questions about the consequences of the plasma interaction that still remain unknown at Mars. $\mathbf{A}$ continuous in-situ solar wind monitor at Mars, together with atmospheric simultaneous observations are needed to fully understand the 3D plasma system, and for having an efficient and continuous space environment monitoring resource.

\section{Conclusions}

The future of Mars science and exploration requires coordinated multi-point space environment and upper atmosphere measurements with sufficient temporal and spatial coverage, as well as solar and solar wind monitoring at Mars, to untangle the complex Martian system from its surface to space. A summary of the science gaps covered in this White Paper and types of missions/payloads that would be appropriate to address them is in Table 1. 
Table 1 : Summary of the current science gaps and types of payload to address them

\begin{tabular}{|c|c|c|c|c|}
\hline Science Gaps & Unknown process & $\begin{array}{l}\text { Ideal type of } \\
\text { mission }\end{array}$ & $\begin{array}{c}\text { Fundamental } \\
\text { payload }\end{array}$ & $\begin{array}{c}\text { Important } \\
\text { payload }\end{array}$ \\
\hline $\begin{array}{l}\text { SCIENCE GAP 1: } \\
\text { Coupled solar wind, } \\
\text { magnetospheric, } \\
\text { ionospheric and } \\
\text { upper atmosphere } \\
\text { dynamics } \\
\text { investigations } \\
\text { (multi-point } \\
\text { observational gaps) }\end{array}$ & $\begin{array}{l}\text {-Solar wind control of } \\
\text { magnetosphere, } \\
\text { plasma boundaries } \\
\text { and currents } \\
\text {-Role of crustal fields } \\
\text {-Neutral \& ion escape } \\
\text {-Plasma transport } \\
\text {-Nightside } \\
\text { precipitation, auroras } \\
\text {-Lower-upper } \\
\text { atmospheric coupling }\end{array}$ & $\begin{array}{l}\text {-Constellation } \\
\text { simultaneous } \\
\text { coordinated } \\
\text { orbiters }\end{array}$ & \multirow{3}{*}{$\begin{array}{l}\text {-Magnetometer } \\
\text {-Electric field } \\
\text {-lon mass } \\
\text { spectrometer } \\
\text { (able to resolve at } \\
\text { least } \mathrm{H}^{+}, \mathrm{He}^{+}, \mathrm{O}^{+}, \\
\mathrm{O}_{2}^{+}, \mathrm{CO}^{+} \text {) } \\
\text {-Neutral mass } \\
\text { spectrometer } \\
\text {-Electron } \\
\text { spectrometer } \\
\text {-Langmuir Probe } \\
\text {-Energetic particle } \\
\text { detector (electron } \\
\text { and protons) } \\
\text {-Solar EUV } \\
\text { monitor } \\
\text {-Energetic Neutral } \\
\text { Analyser (ENA) } \\
\text {-lonospheric radar } \\
\text { (topside and } \\
\text { bottom-side) } \\
\text {-Radiation } \\
\text { monitor }\end{array}$} & \multirow{3}{*}{$\begin{array}{l}\text {-Neutron monitor } \\
\text {-Wind } \\
\text { interferometer } \\
\text {-Radio occultation } \\
\text { with Earth and } \\
\text { between } \\
\text { satellites }{ }^{[46]} \\
\text {-VHF TEC } \\
\text { instrument } \\
\text {-IR and UV } \\
\text { spectroscopy } \\
\text {-LIDAR }\end{array}$} \\
\hline $\begin{array}{l}\text { SCIENCE GAP 2: } \\
\text { The lower } \\
\text { ionosphere } \\
\text { (observational } \\
\text { gaps) }\end{array}$ & $\begin{array}{l}\text {-Bottom-side } \\
\text { ionosphere } \\
\text {-Low limit height of } \\
\text { the ionosphere } \\
\text {-HF radio propagation }\end{array}$ & $\begin{array}{l}\text {-Dual radio- } \\
\text { occultations } \\
\text { between two } \\
\text { orbiters } \\
\text {-Ground } \\
\text { lonospheric } \\
\text { sounding }\end{array}$ & & \\
\hline $\begin{array}{l}\text { SCIENCE GAP 3: } \\
\text { Solar activity } \\
\text { effects on Mars' } \\
\text { system } \\
\text { (observational } \\
\text { gaps) }\end{array}$ & $\begin{array}{l}\text {-Missing a long-term } \\
\text { solar wind monitor } \\
\text { upstream of Mars }\end{array}$ & $\begin{array}{l}\text {-Constellation } \\
\text { coordinated } \\
\text { orbiters, one } \\
\text { always in the } \\
\text { solar wind } \\
\text { - Mars L1 } \\
\text { Spacecraft } \\
\text {-Phobos and } \\
\text { Deimos as } \\
\text { monitoring } \\
\text { platforms }^{[38]}\end{array}$ & & \\
\hline
\end{tabular}

References [1] Halekas et al., 2018 https://doi.org/10.1029/2018JA025866; [2] Jakosky et al., 2015 https://doi.org/10.1126/science. aad0210; [3] Fang X., et al., 2015 https://doi.org/10.1002/2015JA021605; [4] Vaisberg et al., 2018 https://doi.org/10.1002/2018JA025202; [5] Smith et al. 1999 https://doi.org/10.1029/1998JE900024; [6] Montabone et al. 2015 https://doi.org/10.1016/j.icarus.2014.12.034; [7] England et al. 2017 https://doi.org/10.1002/2016JA023475; [8] Chaffin et al., 2018 https://doi.org/10.1029/2018JE005574; [9] Hall et al., 2019 https:// doi.org/10.1029/2018JA026404; [10] Edberg et al., 2009 https://doi.org/10.1016/i.pss.2008.10.016; [11] Harada et al., 2017 https://doi.org/10.1002/2017GL074897; [12]Ermakov et al., 2017 https://doi.org/10.1134/S0038094617050021; [13] Ramstad et al. 2020 https://doi.org/10.1038/s41550-020-1099-y; [14] Jakosky et al. 2015 https://doi.org/10.1126/science.aad3443; [15] Chassefiere and Leblanc, 2014 https://doi.org/10.1016/j.pss.2004.07.002; [16] Jakosky et al., 2017 https://doi.org/10.1126/science.aai7721; [17] Mazelle et al., 2004 https://doi.org/10.1023/B:SPAC.0000032717.98679.d0; [18] Curry et al. 2015; https://doi.org/10.1002/2015GL065304; [19] Luhmann et al. 2017 https://doi.org/10.1002/2016JA023513; [20] Mayyasi et al. 2018 https://doi.org/10.1029/2018GL077727; [21] Fowler et al. 2019 https://doi.org/10.1029/2019JA026550; [22] DiBraccio et al. 2017 https://doi.org/10.1002/2016JA023488; [23] Vaisberg et al. 1976; http://adsabs.harvard.edu/abs/1976spre.conf.1033V; [24] Vaisberg and Smirnov, 1986 https://doi.org/10.1016/02731177(86)90046-3; [25] Withers et al. 2012, https://doi.org/10.1029/2012GL053193; [26] Cao et al. 2019 https://doi.org/10.1029/2019JE 005970; [27] Schneider et al. 2018 https://doi.org/10.1029/2018GL077772; [28] Sánchez-Cano et al. 2019 https://doi.org/10.1029/2018J A026403; [29] Dungey, 1961 https://doi.org/10.1103/PhysRevLett.6.47; [30] Mendillo et al. 2006 https://doi.org/10.1126/science.1122099; [31] Withers et al. 2019 https://doi.org/10.1029/2018JA026266; [32] Pätzold et al. 2005 https://doi.org/10.1126/science.1117755; [33] Molina-Cuberos et al. $2008 \mathrm{https://doi.org/10.1007/s11214-008-9340-5;} \mathrm{[34]} \mathrm{Crismani} \mathrm{et} \mathrm{al.} 2017 \mathrm{https} / / / \mathrm{doi} .0 \mathrm{org} / 10.1038 / \mathrm{ngeo2958F}$; [35] Mendillo et al. 2004 https://doi.org/10.1029/2003RS002933; [36] Fowler et al., 2017 https://doi.org/10.1002/2017GL075189; [37] Guo et al. 2015 https://doi.org/10.1088/0004-637X/810/1/24; [38] Guillaume et al., 2019 https://ui.adsabs.harvard.edu/abs/2019EPSC...13.1221T Labstract; [39]_Grard 1995 https://doi.org/10.1006/icar.1995.1048; [40] Cardnell et al. 2016 https://doi.org/10.1002/2016JE005077; [41] Lee et al., 2017 https://doi.org/10.1002/2016JA023495; [42] Dubinin et al. 2011 https://doi.org/10.1007/s11214-011-9831-7; [43] Jakosky et al. 2018 https://doi.org/10.1016/j.icarus.2018.05.03; [44] Lee et al. 2018 https://doi.org/10.1029/2018GL079162; [45] Crismani et al. 2019 https://doi.org/10.1029/2018JA026251; [46] Ao et al., 2015 https://doi.org/10.1002/2015RS005750; [47] Fang et al., 2020 https://doi.org/10.1029/2019JA026838; [48] Fang et al., 2008 https://doi.org/10.1029/2007JA012736; 